\title{
FALSE BPM READINGS AFFECTING ORBIT FEEDBACK*
}

\author{
H. S. Kang ${ }^{\dagger}$, E. H. Lee, K. M. Ha, J. H. Suh, W. W. Lee, M. H. Chun, Y. C. Kim, J. Y. Huang, T. Y. \\ Lee, J. Choi \\ Pohang Accelerator Laboratory, Pohang 790-784, Korea
}

\section{Abstract}

A slow global orbit feedback (SOFB) is routinely operating in the usual user service operation at PLS. The orbit feedback uses 22 correctors in each plane which have 20-bit capability for the vertical plane and 16-bit capability for the horizontal plane, and the feedback speed is 4 seconds. The orbit stability in RMS was maintained below $1 \mu \mathrm{m}$ in both planes for one hour and $3 \mu \mathrm{m}$ for a 12-hour operation. The BPM chamber movement due to the change of synchrotron radiation heat load mainly limits the SOFB performance. The intensity dependence of BPM electronics is well compensated by a look-up table of BPM.

\section{INTRODUCTION}

The PLS lattice is a triple bend achromat with 12 superperiods. The operating beam energy is $2.5 \mathrm{GeV}$ and the present maximum beam current is $200 \mathrm{~mA}$, which is limited by the available RF power of the RF cavity. In the usual user-service operation SOFB is routinely operating and the electron beam is directly injected from the $2.5-\mathrm{GeV}$ linac twice a day. The orbit stability requirement in position is $20 \mu \mathrm{m}$ in the horizontal plane and $3 \mu \mathrm{m}$ in the vertical plane [1].

The number of BPMs is 9 per superperiod and totally 108 BPMs, and the number of correctors is 70 for each plane with the maximum kick angle of 2-mrad. The power supplies for correctors were all 12-bit and the minimum kick angle of corrector is 1-micro-rad. Among them the correctors in the straight sections were replaced with 20-bit for the vertical plane and 16-bit for the horizontal plane, respectively. With the 20-bit capability the minimum kick angle reduces to 0.0038 $\mu \mathrm{rad}$ and the resultant vertical orbit RMS by this kick would be only $0.025 \mu \mathrm{m}$.

MATLAB is used for SOFB [2]. The orbit feedback algorithm uses the SVD method. The feedback runs every 4 seconds. The present algorithm uses about 75 BPMs and 22 correctors. The number of BPMs for feedback is changing because some BPMs show suspicious behaviours; parasitic movement or large current dependence. The beam current dependence table for BPM electronics is used in the feedback.

The correctors for SOFB are the ones located at the straight sections where the horizontal and vertical beta

\footnotetext{
* Supported by the Ministry of Science and Technology of Korea.

† hskang@postech.ac.kr
}

function are relatively high, which helps effectively correct the orbit change due to insertion device gap change. In order to increase the gap motion speed of insertion devices (EPU and U7) the feedforward correction is incorporated into the feedback with the speed of $5 \mathrm{~Hz}$.
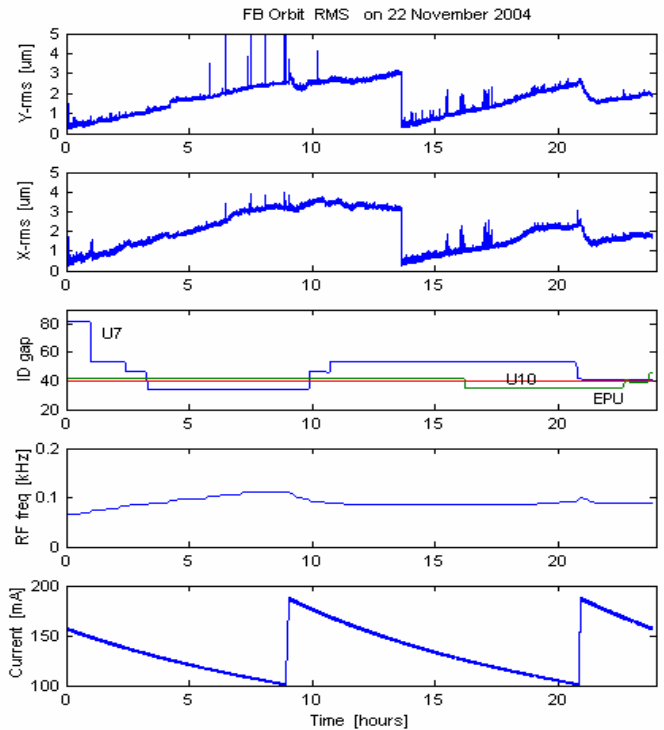

Figure 1: The orbit data for one day under orbit feedback. The first graph: vertical orbit RMS, the second: horizontal orbit RMS, the third: insertion device gap, the fourth: RF frequency, and the last: beam current.

Figure 1 depicts the orbit data for one day under orbit feedback. The vertical orbit RMS is maintained below 3 $\mu \mathrm{m}$ and the horizontal orbit RMS below $4 \mu \mathrm{m}$. The orbit feedback looks like to satisfy the orbit stability requirement. To effectively compensate the BPM electronics' current dependence, the reference orbit is re-set when the beam current reaches $150 \mathrm{~mA}$ after every refill. The current dependence data of BPM electronics is referenced to the BPM reading at $150 \mathrm{~mA}$. However, as shown in Fig. 1, the vertical and horizontal orbit steadily rise from zero where the reference orbit is reset, and get saturated at around $3 \mu \mathrm{m}$. It looks like that the number of correctors is not enough for correction.

Even though the orbit stability is maintained below a few $\mu \mathrm{m}$, just after the current refill we have observed about $10 \mu \mathrm{m}$ vertical position change in a photon BPM at the beamlines. The false reading of BPM makes the actuator in the feedback be driven to the wrong direction. 


\section{FALSE BPM READINGS}

False BPM reading comes from the BPM electronics' dependence on ambient temperature, the BPM electronics' dependence on beam current, and the BPM chamber movement. Compensation of false BPM reading is absolutely necessary in order to minimize the false motion by orbit feedback. The current dependence table is used to subtract the false value from the BPM reading in SOFB. However, so far the chamber movement is not compensated.

The ambient temperature dependence of BPM electronics is difficult to compensate by kind of calibration table, thus should be minimized. The BPM electronics must be influenced by the ambient temperature in the control shed where the BPM electronics locates. One BPM electronics module shows the dependence on ambient temperature of $1.4 \mu \mathrm{m} /{ }^{\circ} \mathrm{C}$. So, the ambient temperature in control shed should be well controlled.

Several BPMs show a very large non-linear intensity dependence which is due to TE mode in antechamber. That BPM electronics uses $375 \mathrm{MHz}$ from the pick-up $\mathrm{RF}$ signal in order to obviate from the TE mode 500 MHz signal.

\section{BPM electronics' dependence on stored beam current}

The BPM system uses the Bergoz MUX-BPM electronics module whose analog position outputs (x any y) are digitised by a VME 16-bit ADC board at 5 kSamples/s.

Figure 2 shows the vertical BPM reading change when the beam is re-filled. This is due to the BPM electronics' dependence on the pickup RF power. The BPM reading changes in RMS between $120 \mathrm{~mA}$ and $180 \mathrm{~mA}$, without including the bad BPMs, are $2.9 \mu \mathrm{m}$ in the horizontal plane and $5.0 \mu \mathrm{m}$ in the vertical plane, which is not small to neglect.

Figure 3 shows the linear change of vertical position reading of 108 BPMs per 1-mA beam current change. This was measured during the beam re-fill. The blue line corresponds to the beam current change between $112 \mathrm{~mA}$ and $153 \mathrm{~mA}$, the green line between $153 \mathrm{~mA}$ and $188 \mathrm{~mA}$, and the red line between $112 \mathrm{~mA}$ and $188 \mathrm{~mA}$. Some BPMs (BPM 1-5, 12-4) show the different rates of position change at the two current ranges: low current rage of $153-112 \mathrm{~mA}$ (blue line), high current range of $188-153 \mathrm{~mA}$ (green line), which means there must be a non-linear current dependence of BPM reading. Neglecting this small non-linearity, we can use the linear change rate from the beam current of 112 to $188 \mathrm{~mA}$ (red line). We set the allowable upper limit at $0.3 \mu \mathrm{m} / \mathrm{mA}$, which corresponds to $30-\mu \mathrm{m}$ reading change with 100-mA current change. The BPMs which has a large change rate such as BPM 3-1 and 5-1 are omitted in the feedback.

We set the reference beam current for the intensity dependence table at $150 \mathrm{~mA}$. The corrector-BPM response matrix essential for orbit feedback is measured at the centre of the intensity dependence table, $150 \mathrm{~mA}$.

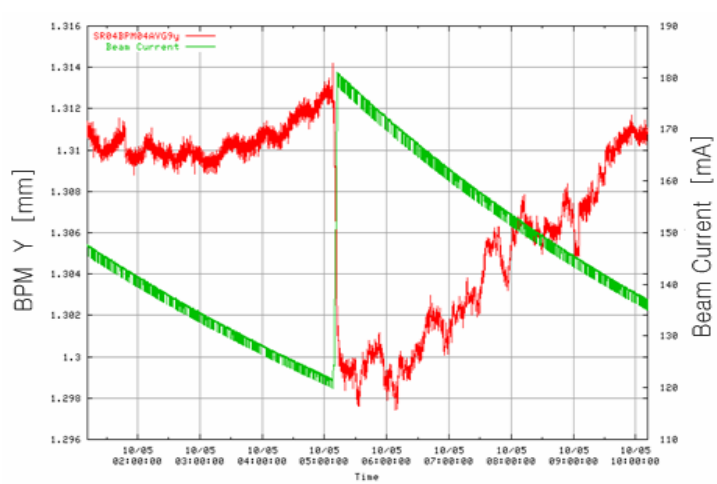

Figure 2: The vertical BPM reading change when the beam is re-filled. The red line: BPM reading, the green line: beam current.

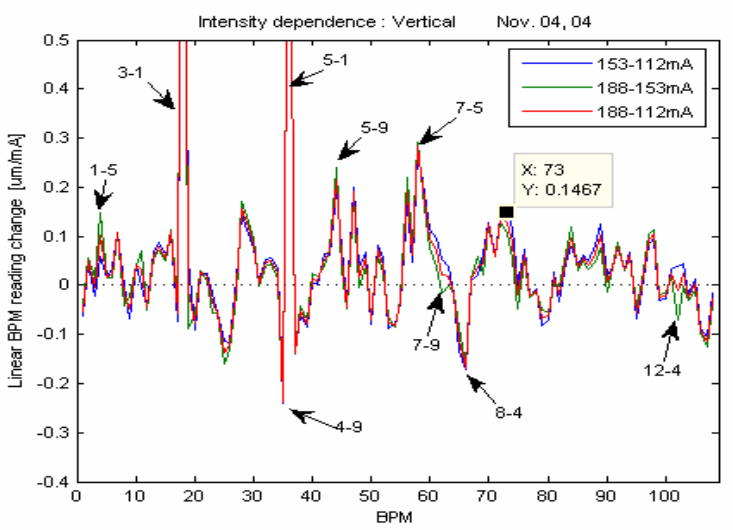

Figure 3: The linear change of vertical position reading of 108 BPMs per 1-mA beam current change. The blue line corresponds to the beam current change between $112 \mathrm{~mA}$ and $153 \mathrm{~mA}$, the green line between $153 \mathrm{~mA}$ and $188 \mathrm{~mA}$, the red line between $112 \mathrm{~mA}$ and $188 \mathrm{~mA}$.

\section{BPM chamber movement}

The vacuum chamber on which BPM is located moves due to the change of synchrotron radiation heat load which is dependent on orbit. Look-up table for compensation is not easy to implement because the chamber motion looks very non-linear and depends on the beam power. And the complexity related to the overall structure exists.

To measure the BPM chamber motion, 6 pairs of linear voltage displacement transducers (LVDT) are installed on the vacuum chamber as shown in Figure 4. The accuracy of the sensor is below $100 \mathrm{~nm}$. Figure 5 shows the measured vacuum chamber motion as the stored beam current changes for one day. The BPM 4-9y and 5-1y shows a change of $10 \mu \mathrm{m}$ after refill and others below $4 \mu \mathrm{m}$. It turns out that the chamber motion strongly depends on the location of chamber support onto girder. In this measurement the beam current changes from $100 \mathrm{~mA}$ to $180 \mathrm{~mA}$. 


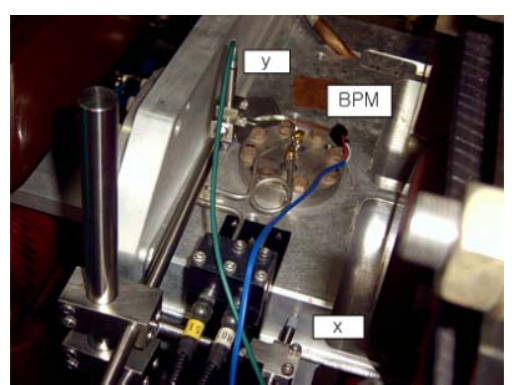

Figure 4: Linear voltage displacement transducers installed on the vacuum chamber near BPM. X denotes the horizontal position sensor and $\mathrm{y}$ the vertical direction.
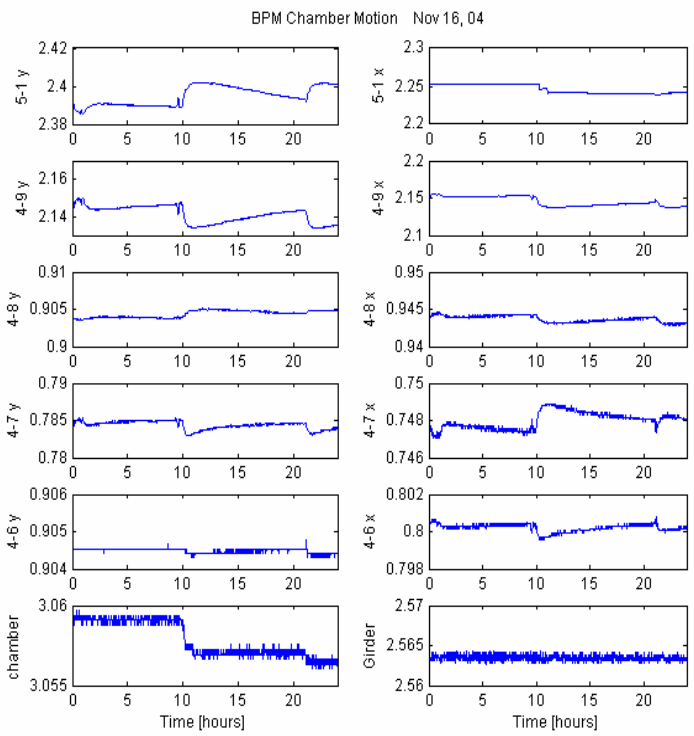

Figure 5: The measured vacuum chamber motion as the stored beam current changes for one day. The vertical scale in each figure is $\mathrm{mm}$. The first number in the name of BPM 5-1y represents the cell number, and the last the BPM number in that cell.

Figure 6 shows the change of corrector current under the orbit feedback for two days. It looks very similar to the BPM chamber movement described in Figure 5. The corrector current rises after the current refill as the chamber position does which means the BPM chamber movement is the major factor of feedback operation. It also looks like that the BPM electronics' current dependence is compensated well.

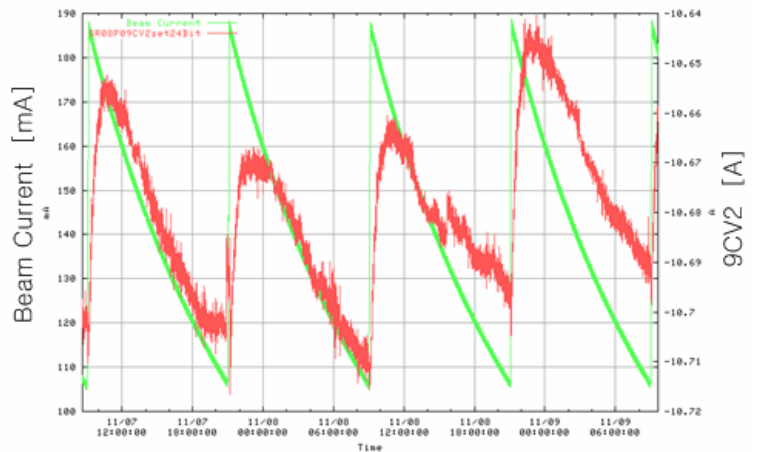

Figure 6: The change of corrector current under the orbit feedback for two days. The red line: corrector (9CV2) current, the green line: beam current.

Real-time measurement of the BPM chamber motion for all BPMs (108 ea) by LVDT will be completed in 2005. The chamber position is monitored with respect to the girder, which is equivalent to quad because the girder is very rigid. The BPM chamber position will be an EPICS database with the data refresh time of 1-3 minutes. In SOFB the data is updated to compensate the chamber motion from the BPM reading. In this correction the girder motion with respect to ground is neglected, and the girder to girder differences also because the quad does not move as the beam current changes. We only care about the orbit with respect to quad.

In 2005, we are replacing all vertical corrector power supplies (70 ea) with fully digital controlled power supply, which out to help improve the SOFB performance.

\section{CONCLUSION}

The achieved orbit stability by SOFB is $<1 \mu \mathrm{m}$ in short term (1 hour) and $<3 \mu \mathrm{m}$ in long term (12 hours). The BPM chamber movement due to synchrotron radiation heating mainly limits the SOFB performance. The intensity dependence of BPM is well compensated by a look-up table of BPM.

\section{ACKNOWLEDGEMENT}

This work was supported by the Ministry of Science and Technology of Korea.

\section{REFERENCES}

[1] H. S. Kang, et al., Proceedings of EPAC 2004, Lucerne, Switzerland, p. 2694

[2] H. S. Kang, et al., Proceedings of ICALEPCS2003, Gyeongju, Korea, p. 160. 